\title{
Prognostic Factors for Patients with Borderline Resectable or Locally Advanced Pancreatic Cancer Receiving Neoadjuvant FOLFIRINOX
}

\author{
Young Hoon Choi ${ }^{1,2}$, Sang Hyub Lee ${ }^{1}$, Min Su You ${ }^{1}$, Bang Sup Shin ${ }^{1}$, Woo Hyun Paik ${ }^{1}$, Ji Kon Ryu ${ }^{1}$, \\ Yong-Tae Kim¹, Wooil Kwon ${ }^{3}$, Jin-Young Jang ${ }^{3}$, and Sun-Whe Kim ${ }^{3}$ \\ ${ }^{1}$ Department of Internal Medicine and Liver Research Institute, Seoul National University Hospital, Seoul National University College \\ of Medicine, ${ }^{2}$ Division of Gastroenterology, Department of Internal Medicine, College of Medicine, The Catholic University of Korea, \\ and ${ }^{3}$ Department of Surgery, Seoul National University Hospital, Seoul National University College of Medicine, Seoul, Korea
}

\section{Article Info}

Received May 28, 2019

Revised December 18, 2019

Accepted February 25, 2020

Published online April 2, 2020

\section{Corresponding Author}

Sang Hyub Lee

ORCID https://orcid.org/0000-0003-2174-9726

E-mail gidoctor@snuh.org
Background/Aims: There has been growing evidence on the utility of neoadjuvant FOLFIRINOX in borderline resectable (BR) or locally advanced (LA) pancreatic cancer. However, factors predicting survival in these patients remain to be identified, and we aimed to identify these prognostic factors.

Methods: Between January 2013 and April 2017, patients with BR or LA pancreatic cancer who received FOLFIRINOX as their initial treatment were identified. Demographic data and clinical outcomes, including the chemotherapy response, conversion to resection, and survival, were reviewed.

Results: A total of 117 patients with $B R(n=39)$ or $L A(n=78)$ pancreatic cancer were included. Of these patients, 29 (24.8\%) underwent curative surgery, and R0 resection was achieved in 21 patients $(72.4 \%)$. The median progression-free survival and overall survival time of all patients were 11.6 and 19.0 months, respectively. In resected patients, the median relapse-free survival and overall survival times were 14.8 and 28.6 months, respectively. In the multivariate Cox model, the lowest level of serum carbohydrate antigen 19-9 (CA 19-9) and resection after FOLFIRINOX were independent factors for improved overall survival. In the subgroup analysis of patients with initial ${ }^{18} \mathrm{~F}$-fluorodeoxyglucose-positron emission tomography (FDG-PET) images, the maximum standardized uptake value (SUVmax) of the pancreatic mass was also shown as an independent factor for improved overall survival.

Conclusions: In patients with BR or LA pancreatic cancer, FOLFIRINOX is a valuable neoadjuvant treatment that enables curative surgery in approximately one-quarter of patients and significantly improves overall survival. In these patients, the prognosis can be estimated using the lowest level of serum CA 19-9, operative status, and initial FDG-PET SUVmax. (Gut Liver $2021 ; 15: 315-323)$

Key Words: Pancreatic neoplasms; Neoadjuvant therapy; FOLFIRINOX; Survival; Prognosis

\section{INTRODUCTION}

Pancreatic ductal adenocarcinoma (PDAC) is a lethal malignancy with a 5 -year survival rate of about $8 \% .{ }^{1}$ Localized PDAC is classified into resectable PDAC, which accounts for approximately $10 \%$ to $15 \%$ of the diagnosed PDAC, and unresectable locally advanced (LA) PDAC, which accounts for $30 \%$ to $35 \%$. $^{1,2}$ For localized PDAC, cases that are potentially resectable but in which $\mathrm{R} 0$ resection is difficult to achieve are called borderline resectable (BR) PDAC., Recently, there has been a debate about which treatment strategy, upfront surgery or neoadjuvant treatment, is better in patients with BR PDAC. To date, a few studies have shown that neoadjuvant treatment has a better prognosis than does upfront surgery. ${ }^{5,6}$ As a result, neoadjuvant treatment rather than surgery has recently 
been preferred in BR PDAC, but the regimen of neoadjuvant treatment has not been specified. Since the introduction of the FOLFIRINOX regimen in 2011, there have been reports of R0 resection after FOLFIRINOX treatment in metastatic PDAC in addition to BR PDAC..$^{7-9}$ Based on these reports, several studies on FOLFIRINOX as a regimen of neoadjuvant treatment have been conducted, and the area of neoadjuvant FOLFIRINOX treatment is gradually expanding from BR PDAC to LA PDAC. ${ }^{10}$ However, most studies on neoadjuvant FOLFIRINOX have focused on efficacy and safety, and there have been few studies on the factors predicting survival. ${ }^{11,12}$

${ }^{18} \mathrm{~F}$-fluorodeoxyglucose-positron emission tomography (FDG-PET) has been reported to be helpful in predicting prognosis in various cancers including PDAC. ${ }^{13-15}$ There is no study to date on whether FDG-PET is helpful in predicting prognosis for patients with BR or LA PDAC who receive neoadjuvant FOLFIRINOX.

Therefore, in this study, we aimed to investigate the predictive factors of overall survival (OS) in patients with $\mathrm{BR}$ or LA PDAC who received neoadjuvant FOLFIRINOX and to investigate the prognostic significance of FDG-PET in subgroup analysis.

\section{MATERIALS AND METHODS}

\section{Patients and data collection}

Patients with BR or LA PDAC who underwent neoadjuvant FOLFIRINOX at the Seoul National University Hospital between January 2013 and April 2017 were included in this study. Resectability of PDAC was evaluated by a multidisciplinary team, including surgeons, radiologists, medical oncologists, and radiation oncologists. Patients who had a history of other malignancy within 5 years or were lost to follow-up were excluded. BR and LA PDAC were defined according to the National Comprehensive Cancer Network criteria. ${ }^{16}$ Demographic and clinical data were obtained from medical records. Variables included age, sex, body mass index, Eastern Cooperative Oncology Group performance status, tumor location, tumor size, node involvement, vascular involvement, FDG-PET maximum standardized uptake value (SUVmax), baseline serum carbohydrate antigen 19-9 (CA 19-9) level, the lowest serum CA 19-9 level during entire FOLFIRINOX cycles including neoadjuvant and palliative treatment, biliary drainage, number of cycles and dose modification of FOLFIRINOX, disease response, surgery, adverse events, and survival.

This study protocol was based on the Declaration of Helsinki and approved by the Institutional Review Board of Seoul National University Hospital (IRB number: 1711107-901). The requirement of informed consent was waived due to the retrospective nature of this study.

\section{Treatment}

We administered the FOLFIRINOX regimen according to the PRODIGE 4/ACCORD 11 trial every 2 weeks. Intravenous infusion of oxaliplatin $85 \mathrm{mg} / \mathrm{m}^{2}$ over 2 hours, immediately followed by intravenous infusion of leucovorin $400 \mathrm{mg} / \mathrm{m}^{2}$ over 2 hours, followed by intravenous infusion of irinotecan $180 \mathrm{mg} / \mathrm{m}^{2}$ over 90 minutes, followed by intravenous bolus of 5 -fluorouracil $400 \mathrm{mg} / \mathrm{m}^{2}$, followed by continuous intravenous infusion of 5 -fluorouracil $2,400 \mathrm{mg} / \mathrm{m}^{2}$ over 46 hours. The dose adjustment or delay according to the patient's performance status or side effects of chemotherapy was done at the discretion of the physician. Relative total dose of chemotherapy, taking into account both dose adjustment and delay, was evaluated using the concept of cumulative relative dose intensity from other study. ${ }^{17}$ The FOLFIRINOX regimen continued to be administered until surgical resection, disease progression, deterioration of performance status, or intolerable toxicity.

\section{Assessment}

Patients visited the hospital for each cycle of chemotherapy and were evaluated for toxicity. Toxicities were assessed according to the National Cancer Institute Common Terminology Criteria for Adverse Events, version 4.03. Tumor responses were evaluated by computed tomography (CT) scans at every three or four cycles of chemotherapy according to the Response Evaluation Criteria in Solid Tumor, version $1.1 .^{18}$ Using these CT scans, resectability of PDAC was evaluated at multidisciplinary team meetings based on the National Comprehensive Cancer Network criteria. ${ }^{16}$ After this resectability evaluation, surgical treatment was performed in the case of resectable PDAC, and in the case of BR PDAC, surgical treatment was carried out according to the decision of the multidisciplinary team. Serum CA 19-9 levels were checked at the time of diagnosis and at every cycle of chemotherapy.

\section{Statistical analysis}

Continuous variables were shown as the median and interquartile range, whereas categorical variables were shown as the number (\%). The cutoff value of FDG-PET SUVmax was chosen as the value that maximized the difference of OS between the two groups that were divided by the cutoff value. This analysis was performed using the $\mathrm{R}$ package maxstat. ${ }^{19}$ Progression-free survival (PFS) was defined as the time from the beginning of FOLFIRINOX to the time of disease progression or death. OS was defined as the time 
from the initiation of FOLFIRINOX to the time of death. Relapse-free survival in resected patients was defined as the time from the operation to the time of recurrence or death. PFS, OS, and relapse-free survival were estimated by the Kaplan-Meier method. The Cox proportional hazard model was used to determine the clinical variables affecting OS. Variables with p-values $<0.1$ in the univariate Cox model were further analyzed by a multivariate Cox model. A p-value $<0.05$ was considered significant. Statistical analyses were performed using SPSS version 23.0 (IBM Corp., Armonk, NY, USA) and R 3.5.1 (The R Foundation for Statistical Computing, Vienna, Austria).

\section{RESULTS}

\section{Patient characteristics}

From January 2013 to April 2017, 137 patients with BR or LA PDAC were treated with FOLFIRINOX. Excluding patients who were lost to follow-up ( $n=18)$ or who had had other cancer within 5 years $(\mathrm{n}=2)$, a total of 117 patients were enrolled in this study. Of these enrolled patients, 48 had initial FDG-PET images (Fig. 1). Baseline characteristics of patients are summarized in Table 1. The median age of patients was 62 years. Most patients (98.3\%) had an Eastern Cooperative Oncology Group performance status of 0-1. At diagnosis, one-third of patients were considered BR (33.3\%) and two-thirds LA (66.7\%). The median serum CA 19-9 level was $470.0 \mathrm{U} / \mathrm{mL}$ (interquartile range, 66.3 to $1,877.0 \mathrm{U} / \mathrm{mL}$ ). Biliary drainage was performed in $24.8 \%$ of the patients.

\section{Response and safety of neoadjuvant FOLFIRINOX}

The median number of entire FOLFIRINOX cycles was seven, and the median number of FOLFIRINOX cycles in 29 patients who underwent surgery after chemotherapy was six. Of the patients, $41.0 \%$ underwent dose reduction from the first cycle, and a total of $60.7 \%$ of patients underwent dose reduction. The median cumulative relative dose intensity was $67.2 \%$. An average of $88.1 \%$ of the full dose was administered to patients. Complete response was achieved in $0.9 \%$ of patients, partial response was achieved in $26.5 \%$ of patients, stable disease was achieved in $63.2 \%$

Table 1. Baseline Characteristics of Patients

\begin{tabular}{|c|c|}
\hline Variable & Value \\
\hline Age, yr & $62(56-67)$ \\
\hline \multicolumn{2}{|l|}{ Sex } \\
\hline Male & $59(50.4)$ \\
\hline Female & $58(49.6)$ \\
\hline Body mass index, $\mathrm{kg} / \mathrm{m}^{2}$ & $22.8(20.7-24.1)$ \\
\hline \multicolumn{2}{|l|}{ ECOG performance status } \\
\hline 0 & $48(41.0)$ \\
\hline 1 & $67(57.3)$ \\
\hline 2 & $2(1.7)$ \\
\hline \multicolumn{2}{|l|}{ Initial imaging modality } \\
\hline CT & $111(94.9)$ \\
\hline MRI & $62(53.0)$ \\
\hline PET & $48(41.0)$ \\
\hline \multicolumn{2}{|l|}{ Tumor location } \\
\hline Head & $58(49.6)$ \\
\hline Body & $51(43.6)$ \\
\hline Tail & $8(6.8)$ \\
\hline Tumor size $(\mathrm{cm})$ & $3.5(2.9-4.6)$ \\
\hline Node involvement & $19(16.2)$ \\
\hline \multicolumn{2}{|l|}{ Vascular involvement } \\
\hline Arterial involvement & 103 (88.0) \\
\hline Venous involvement & $90(76.9)$ \\
\hline \multicolumn{2}{|l|}{ Resectability classification } \\
\hline Borderline resectable & 39 (33.3) \\
\hline Locally advanced & $78(66.7)$ \\
\hline Serum CA 19-9, U/mL & $470.0(66.3-1,877.0)$ \\
\hline Biliary drainage & $29(24.8)$ \\
\hline
\end{tabular}

Data are presented as median (interquartile range) or number (\%). ECOG, Eastern Cooperative Oncology Group; CT, computed tomography; MRI, magnetic resonance imaging; $\mathrm{PET}$, positron emission tomography; CA 19-9, carbohydrate antigen 19-9.

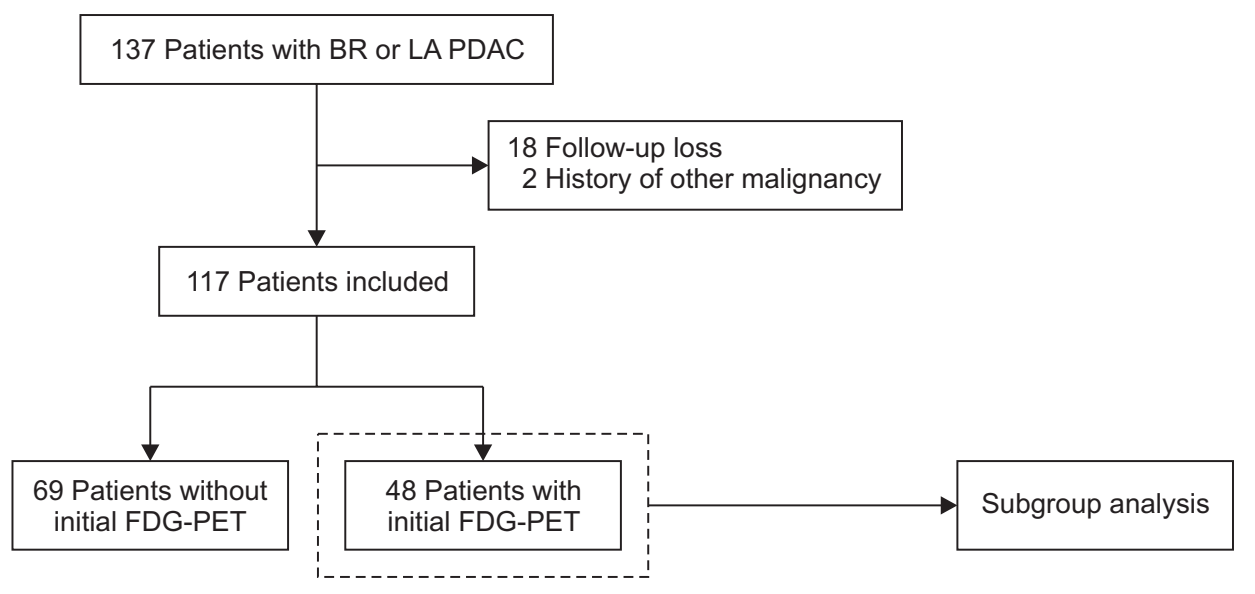

Fig. 1. Flowchart of patient enrollment.

$\mathrm{BR}$, borderline resectable; LA, locally advanced; PDAC, pancreatic ductal adenocarcinoma; FDG-PET, ${ }^{18} \mathrm{~F}$-fluorodeoxyglucose positron emission tomography. 
of patients, and progressive disease was in $4.3 \%$ of patients. As a result, the response rate was $27.4 \%$ and the disease control rate was $90.6 \%$. Five patients $(4.3 \%)$ had progressive disease, four of whom received gemcitabine based second line chemotherapy, and the other received only

Table 2. Summary of FOLFIRINOX Treatment

\begin{tabular}{lc}
\multicolumn{1}{c}{ Variable } & Value \\
\hline Total cycle & $7(4-14)$ \\
Dose reduction & $71(60.7)$ \\
Dose reduction at start & $48(41.0)$ \\
Dose reduction after 1st cycle & $23(19.7)$ \\
Best response & \\
CR & $1(0.9)$ \\
PR & $31(26.5)$ \\
SD & $74(63.2)$ \\
PD & $5(4.3)$ \\
NA & $6(5.1)$ \\
RR (CR+PR) & $32(27.4)$ \\
DCR (CR+PR+SD) & $106(90.6)$ \\
Serum CA 19-9 lowest, U/mL & $51.6(11.0-290.5)$ \\
Resection after FOLFIRINOX & $29(24.8)$ \\
RO & $21 / 29(72.4)$ \\
R1 & $8 / 29(27.6)$ \\
Grade 3 and 4 toxicity & $89(76.1)$ \\
Hematologic & \\
Neutropenia & $73(62.4)$ \\
Febrile neutropenia & $7(6.0)$ \\
Thrombocytopenia & $13(11.1)$ \\
Anemia & $15(12.8)$ \\
Non-hematologic & $23(19.7)$ \\
Nausea and vomiting & $5(4.3)$ \\
Diarrhea & $1(0.9)$ \\
Hypersensitivity & $3(2.6)$ \\
Mucositis & \\
\hline
\end{tabular}

Data are presented as median (interquartile range) or number (\%). $C R$, complete response; PR, partial response; $S D$, stable disease; $P D$, progressive disease; NA, not assessed; RR, response rate; DCR, disease control rate; CA 19-9, carbohydrate antigen 19-9.

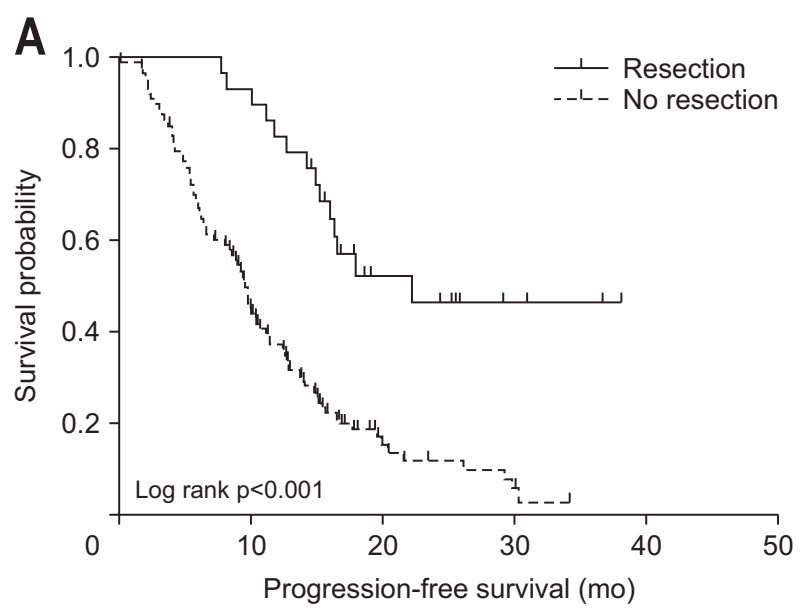

supportive care. The median of the lowest serum CA 19-9 level was $51.6 \mathrm{U} / \mathrm{mL}$. Twenty-nine patients $(24.8 \%)$ underwent surgery after neoadjuvant FOLFIRINOX, and 21 of them (72.4\%) achieved R0 resection (Table 2).

Grade 3 or 4 toxicities associated with FOLFIRINOX occurred in 89 patients (76.1\%). The most common grade 3 or 4 adverse events were neutropenia (62.4\%) and febrile neutropenia, which occurred in $6 \%$ of all patients. For non-hematologic grade 3 or 4 adverse events, nausea/vomiting (19.7\%) was the most common (Table 2).

\section{Progression-free survival and overall survival}

The median follow-up was 16.8 months (interquartile range, 13.0 to 22.9 months). Median PFS and OS of all patients were 11.6 months (95\% confidence interval [CI], 9.3 to 13.9 ) and 19.0 months (95\% CI, 16.6 to 21.5 ), respectively. In resected patients, the median relapse-free survival was 14.8 months (95\% CI, 4.2 to 25.5), median PFS was 22.3 months (95\% CI, 16.1 to not available) and median OS was 28.6 months ( $95 \%$ CI, 27.1 to 30.0). On the other hand, median PFS and OS of patients without resection were 9.7 months (95\% CI, 8.6 to 10.8) and 15.4 months ( $95 \%$ CI, 13.8 to 16), respectively (Table 3, Fig. 2).

Table 3. Survival of Patients

\begin{tabular}{cl}
\hline \multicolumn{1}{c}{ Variable } & Median $(95 \% \mathrm{Cl})$ \\
\hline All patients (n=117) & \\
PFS, mo & $11.6(9.3-13.9)$ \\
OS, mo & $19.0(16.6-21.5)$ \\
Resected patients (n=29) & \\
RFS, mo & $14.8(4.2-25.5)$ \\
PFS, mo & $22.3(16.1-\mathrm{NA})$ \\
OS, mo & $28.6(27.1-30.0)$ \\
\hline
\end{tabular}

$\mathrm{Cl}$, confidence interval; PFS, progression-free survival; OS, overall survival; RFS, relapse-free survival; NA, not available.

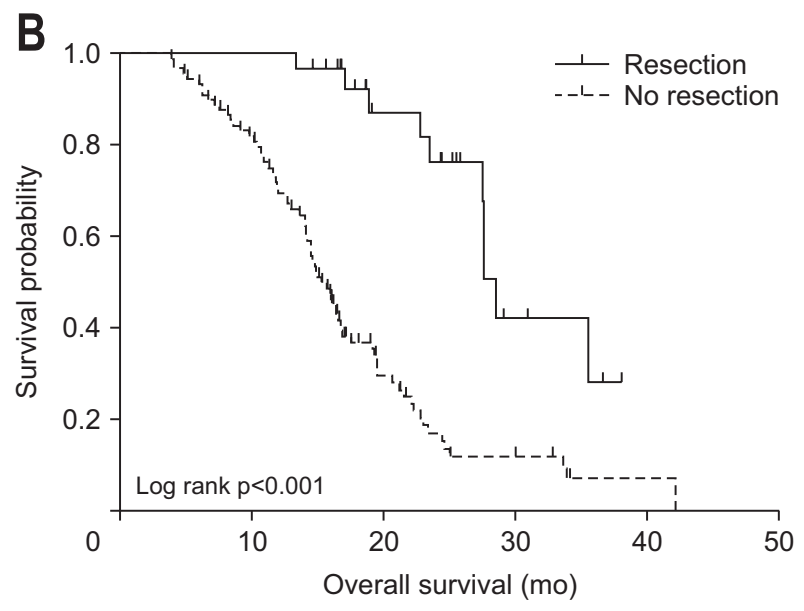

Fig. 2. Progression-free survival (A) and overall survival (B) according to resection. 
Table 4. Univariate and Multivariate Analyses of Factors Associated with Overall Survival by Cox Proportional Hazards Models

\begin{tabular}{|c|c|c|c|c|c|c|}
\hline \multirow{2}{*}{ Variable } & \multicolumn{3}{|c|}{ Univariate } & \multicolumn{3}{|c|}{ Multivariate } \\
\hline & HR & $95 \% \mathrm{Cl}$ & p-value & $\mathrm{HR}$ & $95 \% \mathrm{Cl}$ & $p$-value \\
\hline \multicolumn{7}{|l|}{ Age, yr } \\
\hline$\leq 70$ & 1.000 & & & & & \\
\hline$>70$ & 1.316 & $0.780-2.220$ & 0.303 & & & \\
\hline \multicolumn{7}{|l|}{ Sex } \\
\hline Male & 1.000 & & & & & \\
\hline Female & 1.461 & $0.947-2.256$ & 0.087 & & & \\
\hline \multicolumn{7}{|l|}{ Body mass index, $\mathrm{kg} / \mathrm{m}^{2}$} \\
\hline$\leq 23$ & 1.000 & & & & & \\
\hline$>23$ & 0.802 & $0.517-1.243$ & 0.324 & & & \\
\hline \multicolumn{7}{|l|}{ ECOG performance status } \\
\hline 0 & 1.000 & & & & & \\
\hline 1 and 2 & 0.917 & $0.590-1.425$ & 0.700 & & & \\
\hline \multicolumn{7}{|l|}{ Tumor location } \\
\hline Head & 1.000 & & & & & \\
\hline Body and tail & 0.819 & $0.530-1.265$ & 0.367 & & & \\
\hline \multicolumn{7}{|l|}{ Tumor size, $\mathrm{cm}$} \\
\hline$\leq 3$ & 1.000 & & & & & \\
\hline$>3$ & 1.644 & $1.051-2.569$ & 0.029 & & & \\
\hline \multicolumn{7}{|l|}{ Node involvement } \\
\hline No & 1.000 & & & & & \\
\hline Yes & 0.711 & $0.395-1.281$ & 0.256 & & & \\
\hline \multicolumn{7}{|c|}{ Resectability classification } \\
\hline Borderline resectable & 1.000 & & & & & \\
\hline Locally advanced & 2.332 & $1.390-3.910$ & 0.001 & & & \\
\hline \multicolumn{7}{|c|}{ Serum CA $19-9$ at diagnosis, $\mathrm{U} / \mathrm{mL}$} \\
\hline$\leq 37$ & 1.000 & & & & & \\
\hline$>37$ & 1.580 & $0.853-2.926$ & 0.146 & & & \\
\hline \multicolumn{7}{|l|}{ Biliary drainage } \\
\hline No & 1.000 & & & & & \\
\hline Yes & 1.066 & $0.637-1.783$ & 0.809 & & & \\
\hline \multicolumn{7}{|c|}{ FOLFIRINOX dose reduction } \\
\hline No & 1.000 & & & & & \\
\hline Yes & 1.184 & $0.757-1.853$ & 0.458 & & & \\
\hline \multicolumn{7}{|c|}{ Serum CA 19-9 lowest, U/mL } \\
\hline$\leq 37$ & 1.000 & & & & & \\
\hline$>37$ & 2.445 & $1.545-3.868$ & $<0.001$ & 2.178 & $1.376-3.446$ & 0.001 \\
\hline \multicolumn{7}{|c|}{ Resection after FOLFIRINOX } \\
\hline No & 1.000 & & & & & \\
\hline Yes & 0.213 & $0.109-0.416$ & $<0.001$ & 0.233 & $0.119-0.455$ & $<0.001$ \\
\hline
\end{tabular}

HR, hazard ratio; Cl, confidence interval; ECOG, Eastern Cooperative Oncology Group; CA 19-9, carbohydrate antigen 19-9.

\section{Predictors of overall survival}

In the univariate Cox model, tumor size $>3 \mathrm{~cm}$, LA PDAC, the lowest serum CA 19-9 level $>37 \mathrm{U} / \mathrm{mL}$, and resection after FOLFIRINOX were significantly associated with OS. Multivariate analysis showed that the lowest serum CA 19-9 level >37 U/mL (hazard ratio [HR], 2.178; 95\% CI, 1.376 to 3.446; $\mathrm{p}=0.001$ ) was an independent predictor for poor OS, whereas resection after FOLFIRINOX (HR, 0.233 ; $95 \%$ CI, 0.119 to 0.455 ; $\mathrm{p}<0.001$ ) was an independent predictor for better OS (Table 4).

\section{Cox analysis in subgroup with initial FDG-PET image}

Forty-eight patients (41.0\%) had an initial FDG-PET image. In this subgroup, the univariate Cox model showed that LA PDAC, the lowest serum CA 19-9 level $>37 \mathrm{U} / \mathrm{mL}$, resection after FOLFIRINOX, and FDG-PET SUVmax $>8.4$ were associated with OS. In the multivariate Cox model, the lowest serum CA 19-9 level $>37 \mathrm{U} / \mathrm{mL}$ (HR, 3.674; 95\% CI, 1.549 to 8.715 ; $\mathrm{p}=0.003)$ and FDG-PET SUVmax >8.4 (HR, 2.540; 95\% CI, 1.149 to $5.613 ; \mathrm{p}=0.021$ ) were independent predictors for poor OS, whereas resection after FOLFIRINOX (HR, 0.290; 95\% CI, 0.102 to 
Table 5. Univariate and Multivariate Analyses of Factors Associated with Overall Survival in the Subgroup with Initial FDG-PET Images by Cox Proportional Hazards Models

\begin{tabular}{|c|c|c|c|c|c|c|}
\hline \multirow{2}{*}{ Variable } & \multicolumn{3}{|c|}{ Univariate } & \multicolumn{3}{|c|}{ Multivariate } \\
\hline & $\mathrm{HR}$ & $95 \% \mathrm{Cl}$ & $p$-value & $\mathrm{HR}$ & $95 \% \mathrm{Cl}$ & $\mathrm{p}$-value \\
\hline \multicolumn{7}{|l|}{ Age, yr } \\
\hline$\leq 70$ & 1.000 & & & & & \\
\hline$>70$ & 1.244 & $0.504-3.067$ & 0.635 & & & \\
\hline \multicolumn{7}{|l|}{ Sex } \\
\hline Male & 1.000 & & & & & \\
\hline Female & 1.099 & $0.547-2.206$ & 0.791 & & & \\
\hline \multicolumn{7}{|l|}{ Body mass index, $\mathrm{kg} / \mathrm{m}^{2}$} \\
\hline$\leq 23$ & 1.000 & & & & & \\
\hline$>23$ & 0.611 & $0.292-1.279$ & 0.191 & & & \\
\hline \multicolumn{7}{|c|}{ ECOG performance status } \\
\hline 0 & 1.000 & & & & & \\
\hline 1 and 2 & 0.663 & $0.322-1.366$ & 0.266 & & & \\
\hline \multicolumn{7}{|l|}{ Tumor location } \\
\hline Head & 1.000 & & & & & \\
\hline Body and tail & 0.647 & $0.316-1.326$ & 0.234 & & & \\
\hline \multicolumn{7}{|l|}{ Tumor size, cm } \\
\hline$\leq 3$ & 1.000 & & & & & \\
\hline$>3$ & 1.386 & $0.688-2.793$ & 0.361 & & & \\
\hline \multicolumn{7}{|l|}{ Node involvement } \\
\hline No & 1.000 & & & & & \\
\hline Yes & 0.882 & $0.303-2.563$ & 0.817 & & & \\
\hline \multicolumn{7}{|c|}{ Resectability classification } \\
\hline Borderline resectable & 1.000 & & & & & \\
\hline Locally advanced & 2.816 & $1.321-6.005$ & 0.007 & & & \\
\hline \multicolumn{7}{|c|}{ Serum CA $19-9$ at diagnosis, $\mathrm{U} / \mathrm{mL}$} \\
\hline$\leq 37$ & 1.000 & & & & & \\
\hline$>37$ & 1.513 & $0.577-3.971$ & 0.400 & & & \\
\hline \multicolumn{7}{|l|}{ Biliary drainage } \\
\hline No & 1.000 & & & & & \\
\hline Yes & 1.214 & $0.538-2.740$ & 0.640 & & & \\
\hline \multicolumn{7}{|c|}{ FOLFIRINOX dose reduction } \\
\hline No & 1.000 & & & & & \\
\hline Yes & 1.415 & $0.609-3.291$ & 0.419 & & & \\
\hline \multicolumn{7}{|c|}{ Serum CA 19-9 lowest, U/mL } \\
\hline$\leq 37$ & 1.000 & & & & & \\
\hline$>37$ & 3.114 & $1.385-7.001$ & 0.006 & 3.674 & $1.549-8.715$ & 0.003 \\
\hline \multicolumn{7}{|c|}{ Resection after FOLFIRINOX } \\
\hline No & 1.000 & & & & & \\
\hline Yes & 0.202 & $0.076-0.539$ & 0.001 & 0.290 & $0.102-0.825$ & 0.020 \\
\hline \multicolumn{7}{|l|}{ FDG-PET SUVmax } \\
\hline$\leq 8.4$ & 1.000 & & & & & \\
\hline$>8.4$ & 3.096 & $1.516-6.322$ & 0.002 & 2.540 & $1.149-5.613$ & 0.021 \\
\hline
\end{tabular}

HR, hazard ratio; $\mathrm{Cl}$, confidence interval; ECOG, Eastern Cooperative Oncology Group; CA 19-9, carbohydrate antigen 19-9; FDG-PET, ${ }^{18}$ F-fluorodeoxyglucose positron emission tomography; SUVmax, maximum standardized uptake value.

$0.825 ; \mathrm{p}=0.020)$ was an independent predictor for better OS (Table 5).

\section{DISCUSSION}

Recently, neoadjuvant FOLFIRINOX treatment has been increasingly used, but the factors predicting survival in patients with BR or LA PDAC treated with neoadjuvant FOLFIRINOX have not been well known. This study showed that surgical resection after FOLFIRINOX and the lowest level of serum CA 19-9 were predictors of OS in patients with BR or LA PDAC who were receiving neoadjuvant FOLFIRINOX. Subgroup analysis showed that the initial FDG-PET SUVmax was also an independent predictor of OS. 
In our study, median PFS and OS were 11.6 months and 19.0 months, respectively. These values were within the range of PFS (3.0 to 20.4 months) and OS (10.0 to 32.7 months) of the previous studies on BR or LA PDAC treated with FOLFIRINOX. ${ }^{20}$ Resection was performed in $24.8 \%$ of the patients in this study, of which $72.4 \%$ achieved R0 resection, which was similar to the resection rate of $28 \%$ and the $\mathrm{R} 0$ resection rate of $77 \%$ in the previous studies. ${ }^{21}$ Grade 3 or 4 toxicities associated with FOLFIRINOX were present in $76.1 \%$ of total patients and were higher than in previous studies. In particular, neutropenia was $62.4 \%$, which was higher than that of previous studies by about $20 \% .{ }^{22,23}$ However, similar or higher rates of grade 3 or 4 neutropenia in our study can be seen in other studies related to FOLFIRINOX conducted in Korea and Japan. ${ }^{11,24}$ Considering that the aforementioned studies, which had relatively low neutropenia rates, were conducted in the west, it can be assumed that Asian people are more vulnerable to bone marrow suppression by FOLFIRINOX treatment. Despite the high incidence of neutropenia in this study, febrile neutropenia accounted for $6 \%$ of all patients and no deaths associated with neutropenia occurred.

CA 19-9 is a well-known tumor marker of pancreatic cancer. ${ }^{25}$ However, the prognostic value of the lowest level of CA 19-9 during chemotherapy was not well investigated. Previous studies on patients receiving chemotherapy in pancreatic cancer have rarely dealt with the lowest level of CA 19-9 and have focused primarily on the association between survival and the degree of CA 19-9 decrease after chemotherapy. ${ }^{26,27}$ In the previous studies, the criteria for decreased CA $19-9$ ranged from $15 \%$ to $89 \%$ based on the values measured after six to eight weeks of chemotherapy. ${ }^{26,27}$ In this study, the degree of decrease of CA 19-9 after three or four cycles of FOLFIRINOX was divided by various criteria, such as $20 \%, 25 \%, 50 \%$, and $75 \%$, and the association with the OS was investigated (data not shown). Among these criteria, the criterion of $75 \%$ or more decrease of CA 19-9 after three cycles of FOLFIRINOX showed the lowest $\mathrm{p}$-value $(\mathrm{p}=0.072)$ in the univariate Cox analysis of OS, but it was not statistically significant. In contrast, the lowest level of CA 19-9 in the same analysis was statistically significant. These results suggest that the lowest level of CA 19-9 may be more related to prognosis than is the degree of CA 19-9 decrease. However, further studies are needed, because there is little research on the association between survival and the lowest level of CA 19-9 in patients receiving chemotherapy.

Another predictor of OS in this study is whether to perform resection after FOLFIRINOX treatment. Of the 29 patients who underwent surgery after FOLFIRINOX treatment, the response to chemotherapy was mostly par- tial response $(48.3 \%)$ or stable disease $(48.3 \%)$, and one patient had complete response (3.4\%) and no progressive disease. The resectability classifications of these 29 patients by preoperative CT were resectable in seven patients, BR in 21 patients, and unresectable in one patient. The patient with the pancreatic lesion classified as unresectable had no hypermetabolic lesion on preoperative FDG-PET, and it was judged that we could not distinguish whether the entire pancreatic lesion observed on preoperative CT was the true malignant lesion. This patient was relatively young, age 45 years, and underwent surgical resection for curative treatment and achieved $\mathrm{R} 0$ resection. This result is in line with previous studies showing that CT imaging after neoadjuvant FOLFIRINOX treatment does not accurately predict resectability. ${ }^{28,29}$ Therefore, BR or LA PDAC that has not obviously progressed during FOLFIRINOX treatment should be considered for surgery if possible, and additional investigation such as FDG-PET may be helpful in decision making. Further studies are needed to find out which cases are more suitable for curative surgery.

Subgroup analysis also showed that initial FDG-PET SUVmax $>8.4$ is a predictor of a worse prognosis. Several studies on pancreatic cancer and FDG-PET have shown that the cutoff value is slightly different for each study, but as in our study, the higher the SUVmax of the initial PET, the worse the prognosis. ${ }^{30}$ To our knowledge, this is the first study to show FDG-PET SUVmax to be a prognostic factor in patients with BR or LA PDAC treated with FOLFIRINOX. In addition, the lowest level of CA 19-9, and surgical resection were still significant predictors of OS in the subgroup analysis, although the number of subjects in this subgroup analysis was significantly reduced to 48 .

This study has several limitations. First, this is a retrospective study in a single institution. Second, we used only the SUVmax value of the initial FDG-PET image rather than the change of the SUVmax value because follow-up FDG-PET images were rarely done. In addition, it is difficult to draw the prognostic value of SUVmax due to the relatively small number of patients who underwent initial FDG-PET image. Nevertheless, it is meaningful that we first found that the initial FDG-PET SUVmax value was associated with OS in patients with BR or LA PDAC treated with FOLFIRINOX.

In conclusion, our study showed that the lowest level of serum CA 19-9 >37 U/mL and the value of initial FDGPET SUVmax $>8.4$ were independent predictors of poor prognosis, whereas surgical resection after FOLFIRINOX was an independent predictor of longer OS. Considering these results, surgical treatment should be considered more actively in patients with BR or LA PDAC treated with FOLFIRINOX. If serum CA 19-9 level does not fall below 
$37 \mathrm{U} / \mathrm{mL}$ during FOLFIRINOX treatment, or if the initial FDG-PET SUVmax is greater than 8.4, more aggressive treatment and short-term follow-up can be considered.

\section{CONFLICTS OF INTEREST}

No potential conflict of interest relevant to this article was reported.

\section{ACKNOWLEDGEMENTS}

This article was presented at the 2nd Korea Digestive Disease Week (KDDW 2018) on November 16, 2018, in Seoul, Korea.

\section{AUTHOR CONTRIBUTIONS}

Study concept and design: S.H.L., Y.H.C. Data acquisition: M.S.Y., B.S.S. Data analysis and interpretation: Y.H.C., W.H.P., W.K., J.K.R., Y.T.K., J.Y.J., S.W.K. Drafting of the manuscript: Y.H.C. Critical revision of the manuscript: S.H.L., J.K.R., Y.T.K., J.Y.J., S.W.K.

\section{ORCID}

Young Hoon Choi https://orcid.org/0000-0002-2633-1401 Sang Hyub Lee https://orcid.org/0000-0003-2174-9726 Min Su You https://orcid.org/0000-0001-8775-6131 Bang Sup Shin https://orcid.org/0000-0002-3702-6760 Woo Hyun Paik https://orcid.org/0000-0001-8708-3280 Ji Kon Ryu Yong-Tae Kim Wooil Kwon Jin-Young Jang Sun-Whe Kim https://orcid.org/0000-0001-8798-0491 https://orcid.org/0000-0002-4842-6874 https://orcid.org/0000-0002-4827-7805 https://orcid.org/0000-0003-3312-0503 https://orcid.org/0000-0001-6315-6019

\section{REFERENCES}

1. Siegel RL, Miller KD, Jemal A. Cancer Statistics, 2017. CA Cancer J Clin 2017;67:7-30.

2. Heinemann V, Boeck S. Perioperative management of pancreatic cancer. Ann Oncol 2008;19 Suppl 7:vii273- vii278.

3. Stathis A, Moore MJ. Advanced pancreatic carcinoma: current treatment and future challenges. Nat Rev Clin Oncol 2010;7:163-172.

4. Ryan DP, Hong TS, Bardeesy N. Pancreatic adenocarcinoma.
N Engl J Med 2014;371:1039-1049.

5. Versteijne E, Vogel JA, Besselink MG, et al. Meta-analysis comparing upfront surgery with neoadjuvant treatment in patients with resectable or borderline resectable pancreatic cancer. Br J Surg 2018;105:946-958.

6. Jang JY, Han Y, Lee H, et al. Oncological benefits of neoadjuvant chemoradiation with gemcitabine versus upfront surgery in patients with borderline resectable pancreatic cancer: a prospective, randomized, open-label, multicenter phase 2/3 trial. Ann Surg 2018;268:215-222.

7. Conroy T, Desseigne F, Ychou M, et al. FOLFIRINOX versus gemcitabine for metastatic pancreatic cancer. N Engl J Med 2011;364:1817-1825.

8. Schneitler S, Kröpil P, Riemer J, et al. Metastasized pancreatic carcinoma with neoadjuvant FOLFIRINOX therapy and R0 resection. World J Gastroenterol 2015;21:6384-6390.

9. Gostimir M, Bennett S, Moyana T, Sekhon H, Martel G. Complete pathological response following neoadjuvant FOLFIRINOX in borderline resectable pancreatic cancer: a case report and review. BMC Cancer 2016;16:786.

10. Murphy JE, Wo JY, Ryan DP, et al. Total neoadjuvant therapy with FOLFIRINOX followed by individualized chemoradiotherapy for borderline resectable pancreatic adenocarcinoma: a phase 2 clinical trial. JAMA Oncol 2018;4:963-969.

11. Yoo C, Kang J, Kim KP, et al. Efficacy and safety of neoadjuvant FOLFIRINOX for borderline resectable pancreatic adenocarcinoma: improved efficacy compared with gemcitabine-based regimen. Oncotarget 2017;8:46337-46347.

12. Boone BA, Steve J, Krasinskas AM, et al. Outcomes with FOLFIRINOX for borderline resectable and locally unresectable pancreatic cancer. J Surg Oncol 2013;108:236-241.

13. Ma W, Wang M, Li X, et al. Quantitative (18)F-FDG PET analysis in survival rate prediction of patients with nonsmall cell lung cancer. Oncol Lett 2018;16:4129-4136.

14. Diao W, Tian F, Jia Z. The prognostic value of SUV(max) measuring on primary lesion and ALN by (18)F-FDG PET or PET/CT in patients with breast cancer. Eur J Radiol 2018;105:1-7.

15. Lee JW, Kang CM, Choi HJ, et al. Prognostic value of metabolic tumor volume and total lesion glycolysis on preoperative ${ }^{18} \mathrm{~F}$-FDG PET/CT in patients with pancreatic cancer. J Nucl Med 2014;55:898-904.

16. Tempero MA, Malafa MP, Al-Hawary M, et al. Pancreatic Adenocarcinoma, Version 2.2017, NCCN Clinical Practice Guidelines in Oncology. J Natl Compr Canc Netw 2017;15:1028-1061.

17. Lee JC, Kim JW, Ahn S, et al. Optimal dose reduction of FOLFIRINOX for preserving tumour response in advanced pancreatic cancer: using cumulative relative dose intensity. Eur J Cancer 2017;76:125-133.

18. Eisenhauer EA, Therasse P, Bogaerts J, et al. New response 
evaluation criteria in solid tumours: revised RECIST guideline (version 1.1). Eur J Cancer 2009;45:228-247.

19. Lausen B, Schumacher M. Maximally selected rank statistics. Biometrics 1992;48:73-85.

20. Suker M, Beumer BR, Sadot E, et al. FOLFIRINOX for locally advanced pancreatic cancer: a systematic review and patient-level meta-analysis. Lancet Oncol 2016;17:801-810.

21. Rombouts SJ, Walma MS, Vogel JA, et al. Systematic review of resection rates and clinical outcomes after FOLFIRINOXbased treatment in patients with locally advanced pancreatic cancer. Ann Surg Oncol 2016;23:4352-4360.

22. Faris JE, Blaszkowsky LS, McDermott $S$, et al. FOLFIRINOX in locally advanced pancreatic cancer: the Massachusetts General Hospital Cancer Center experience. Oncologist 2013;18:543-548.

23. Hosein PJ, Macintyre J, Kawamura C, et al. A retrospective study of neoadjuvant FOLFIRINOX in unresectable or borderline-resectable locally advanced pancreatic adenocarcinoma. BMC Cancer 2012;12:199.

24. Todaka A, Mizuno N, Ozaka M, et al. Nationwide multicenter observational study of FOLFIRINOX chemotherapy in 399 patients with unresectable or recurrent pancreatic cancer in Japan. Pancreas 2018;47:631-636.

25. Boeck S, Stieber P, Holdenrieder S, Wilkowski R, Heine- mann V. Prognostic and therapeutic significance of carbohydrate antigen 19-9 as tumor marker in patients with pancreatic cancer. Oncology 2006;70:255-264.

26. Ballehaninna UK, Chamberlain RS. The clinical utility of serum CA 19-9 in the diagnosis, prognosis and management of pancreatic adenocarcinoma: an evidence based appraisal. J Gastrointest Oncol 2012;3:105-119.

27. Robert M, Jarlier M, Gourgou S, et al. Retrospective analysis of CA19-9 decrease in patients with metastatic pancreatic carcinoma treated with FOLFIRINOX or gemcitabine in a randomized phase III study (ACCORD11/PRODIGE4). Oncology 2017;93:367-376.

28. Ferrone CR, Marchegiani G, Hong TS, et al. Radiological and surgical implications of neoadjuvant treatment with FOLFIRINOX for locally advanced and borderline resectable pancreatic cancer. Ann Surg 2015;261:12-17.

29. Wagner M, Antunes C, Pietrasz D, et al. CT evaluation after neoadjuvant FOLFIRINOX chemotherapy for borderline and locally advanced pancreatic adenocarcinoma. Eur Radiol 2017;27:3104-3116.

30. Zhu D, Wang L, Zhang H, et al. Prognostic value of $18 \mathrm{~F}-$ FDG-PET/CT parameters in patients with pancreatic carcinoma: a systematic review and meta-analysis. Medicine (Baltimore) 2017;96:e7813. 